\title{
3 Research Square \\ The Role of Posterior Pallial Amygdala in Mediating Motor Behaviors in Pigeons
}

\section{Xinmao Tian}

Zhengzhou University

Yuhua Shi

Zhengzhou University

Yifeng Zhang

Zhengzhou University

Yijie Wang

Zhengzhou University

Mengke Li

Zhengzhou University

Han Cheng

Zhengzhou University

Zhenlong Wang ( $\square$ wzl@zzu.edu.cn )

Zhengzhou University

\section{Research Article}

Keywords: posterior pallial amygdala (PoA) , mediating motor behaviors , pigeons, Columba livia

Posted Date: August 10th, 2021

DOI: https://doi.org/10.21203/rs.3.rs-770996/v1

License: (c) (i) This work is licensed under a Creative Commons Attribution 4.0 International License.

Read Full License

Version of Record: A version of this preprint was published at Scientific Reports on January 10th, 2022. See the published version at https://doi.org/10.1038/s41598-021-03876-7. 


\section{Abstract}

The posterior pallial amygdala (PoA) is located on the basolateral caudal telencephalon, including the basal division of PoA (PoAb) and the compact division of PoA (PoAc). PoA plays a vital role in emotion regulation and is considered a part of the amygdala in birds. However, the regulatory functions responsible for motor behaviors and emotions between PoAb and PoAc are poorly understood. Therefore, we studied the structure and function of PoA by tract-tracing methods, constant current electrical stimulation, and different dopamine receptor drug injections in pigeons (Columba livia). PoAb connects reciprocally with two nuclear groups in the cerebrum: 1) a continuum comprising the temporo-parietooccipitalis, corticoidea dorsolateralis, hippocampus, and parahippocampalis areas and 2) rostral areas of the hemisphere, including the nucleus septalis lateralis and nucleus taeniae amygdalae.

Extratelencephalic projections of PoAb terminate in the lateral hypothalamic nucleus and are scattered in many limbic midbrain regions. PoAb and PoAc mainly mediated the turning movement. D1 agonist and D2 antagonist could significantly reduce the latency period for entering into the central area and increase the stay time in the central area, whereas D1 antagonist and D2 agonist had the opposite effect. PoAb and PoAc are important brain areas that mediate turning behavior.

\section{Introduction}

The arcopallium/posterior pallial amygdala complex, previously known as the archistriatum (A), is present in the avian posterior ventral telencephalon ${ }^{1}$. After the Avian Brain Nomenclature Forum held at Duke University in July 2002, the Forum renamed most of the structures in the archistriatum that shared premotor characteristics to arcopallium, and for the remaining parts of the archistriatum that were assumed to constitute the amygdala, the Forum appended amygdala to their names to distinguish them from the arcopallial nuclei ${ }^{1}$. The acropallium/posterior pallial amygdala complex plays an essential role in controlling fear reactions in avians ${ }^{2-6}$. However, limited information is available about the contribution of its subdivisions.

As parts of the acropallium/posterior pallial amygdala complex, the posterior pallial amygdala (PoA) comprises the basal division of PoA (PoAb) and the compact division of PoA (PoAc). At present, PoAc shows some similarities in the receptor expression profile of the mammalian lateral regions of the amygdala ${ }^{7}$. However, there are few studies on PoAb ${ }^{7-9}$, and the role of PoAb and PoAc in mediating motor behaviors of birds remains unknown. Hence, these issues will be discussed in the present study.

As a common bird in daily lives, homing pigeons (Columba livia domestica) have many outstanding abilities, including homing and good orientation discrimination, making them an ideal model for studying neurological mechanisms. In this study, we defined the afferent and efferent projections of PoAb in the pigeon using cholera toxin subunit B (CTB) and biotinylated dextran amine (BDA). Subsequently, we investigated the effects of PoA on the mediation of motor behavior in pigeons via electrical stimulation. Lastly, we also tested the effects of different dopamine D1 and D2 receptor agonists and antagonists on forward behavior. 


\section{Results}

\section{Fiber connections of PoAb}

Retrograde labeling by CTB. The center of the injection site of the right PoAb was placed at A 6.25, L 7.6, and $D 6.8$ according to the atlas of Pigeon ${ }^{13}$, and the diffusion of the tracer was mostly restricted within PoAb (Figure 1b). Labeled areas outside PoAb included many regions of the forebrain. Around PoAb, numerous labelings were found in the cortex piriformis (CPI) from approximately A 5.00 to A 7.50, fewer labelings were found in the arcopallium dorsale (AD) and arcopallium intermedium (Al), and sparse labelings were found in the arcopallium anterius (AA) (Figure $2 c-f$ ). Other labeled areas outside PoAb were divided into three streams in the telencephalon. Two of them were from the nidopallium caudoventrale, in which an extensively labeled area was found to be separated. One of them traveled dorsolaterally, along the way through the parahippocampalis (APH) and hippocampus $(\mathrm{Hp})$ areas to the dorsolateral corticoid (CDL) and temporo-parieto-occipitalis (TPO) areas (Figure 2c$\mathrm{g}$ ); in contrast, the other ran ventrally and turned rostrally, passing through the nidopallium caudolaterale (NCL), dorsal mesopallium (MD), and ventral mesopallium (MV). Labeled neurons proceeding rostrally extended to the hyperpallium apicale $(\mathrm{HA})$, hyperpallium densocellulare, hyperpallium ventrale $(\mathrm{HV})$, and hyperpallium intercalatum $(\mathrm{HI})$ of the rostral telencephalon (Figure 2a-c). They converged in the pallium at about A 7.0. The third course ran basal and turned rostrally. En route, it branched to nucleus taeniae amygdalae (TnA), nucleus septalis lateralis (SL) and the anterior commissura (AC). Labeled neurons proceeding further rostrally extended to the basorostral pallial nucleus.

Numerous labeled neurons were observed in the nucleus dorsomedialis anterior thalami (DMA) (Figure $2 d-f)$ of the diencephalon. In contrast, fewer labeled neurons were observed in the radix mesencephalicus nervi trigemini (RxVM) (Figure 1e, Figure 2i), tectum opticum (TeO) (Figure 2e) and nucleus preopticus anterior (POA). Sparsely distributed labeled neurons were observed in the lateral hypothalamic nucleus (LHy) (Figure 2c) and nucleus rotundus.

In the midbrain, labeled neurons were widely scattered to a different site. CTB-labeled neurons were distributed in a continuous region stratum griseum central (SGC), stratum griseum et fibrosum superficiale (SGF) (Figure 1c-e, Figure 2g-i), nucleus isthmi, pars magnocellularis (Imc) (Figure 1c-d), nucleus isthmi, pars parvocellularis (Ipc) (Figure 1c-d), and formatio reticularis medialis mesencephali. Fewer labeled neurons were observed in the substantia grisea centralis (GCt) (Figure $2 g-h$ ), nucleus intercollicularis (ICo) (Figure $2 g-h$ ), substantia grisea et fibrosa periventricularis (SGP). Sparse labeling was observed in the locus ceruleus (LOC), nucleus pontis lateralis (PL), stratum album central (SAC) (Figure 1c-e, Figure $2 \mathrm{~h}-\mathrm{i}$ ), nucleus subpretectalis (SP) (Figure 2f), and ventral tegmental area (VTA).

Anterograde labeling by BDA. The injection of BDA to the right PoAb was located at A 5.75, L 7.60, D 6.10, and diffusion of the tracer was restricted within the nucleus (Figure 1g). Similar to CTB-labeled neurons, around PoAb, many BDA-labeled fibers were found in CPI, AD, and AI. Moderate numbers of BDA- 
labeled fibers were observed in AA and arcopallium mediale (Figure 2c-f). In contrast, PoAc contained few labeled fibers. Rostral to the injection, projections from PoAb were divided into four streams: one traveled dorsolaterally of the forebrain, along the way through APH and Hp to CDL and TPO, and among them, CDL extensively contained BDA-labeled fibers (Figure 2c-g); in contrast, the other ran ventrally and turned rostrally, and the BDA-labeled fibers forming the continuum were found to exist extensively throughout NCL, MD, and MV. Labeled fibers extended to the HA, proceeding further rostrally. Less numerous labeled fibers existed in HV, and very sparsely labeled areas were observed in $\mathrm{HI}$ of the rostral telencephalon (Figure 2a-c). The first two streams joined in the pallium, similar to the findings observed for CTB labeling. The third stream ascending fiber bundle extended rostrally toward the basal forebrain, and on this pathway, many fibers appeared in TnA (Figure 2e). Moderate numbers of fibers appeared in nucleus septalis lateralis and tuberculum olfactorium, and more rostrally, terminated in $A C$ at approximately A 7.75. Using AC as a turning point, the contralateral bundle repeated its ipsilateral in a mirror or symmetric fashion. The fourth stream ran through the diencephalon and midbrain via a descending fiber bundle.

In the diencephalon, the main course of the descending pathway was primarily through the following areas: DMA and DMP in the rostral diencephalon (Figure 2e-f); then TeO and POM in the caudal diencephalon; and finally, a limited number of fibers in nIV, LHy, and RxVM.

In the midbrain, the BDA-labeled areas were much more than the CTB-labeled areas. The descending pathway labeled many areas along its course, and the labeled areas were divided into two streams. Ventrally, it projected massively to the limbic midbrain. There were several labeled varicose fibers or terminals in fasciculus longitudinalis medialis, SGC, SGF, and SP (Figure $1 \mathrm{~h}-\mathrm{j}$, Figure $2 \mathrm{~g}-\mathrm{i}$ ). In addition, BDA-labeled neurons were frequently observed in GCt, SAC, SGP, and Sop (Figure $2 \mathrm{~g}-\mathrm{i}$ ). The other labeled fibers were scattered in the midbrain, and these areas received a moderate number of labeled fibers from the descending pathway, including Imc, Ipc, OM and LoC (Figure 1j). Few labeled fibers were distributed sparsely in torus semicircularis, VTA, and tractus vestibule-mesencephalicus.

In the CTB and BDA injections, three pigeons each were were targeted to PoAb. In each case, two were largely confined to the PoAb and with successful labeling, so they were selected to describe and depict the results. And the summary Figures and Table 1 represent composites results. The general patterns of the afferent and efferent projections of PoAb are summarized in Figure 3 and Table 1.

Table 1

Distribution of labeling of BDA and CTB injection in PoAb 
Number of CTB - labeled neurons

Sites

Telencephalon

AA (arcopallium anterius)

AC (anterior commissura)

AD (arcopallium dorsale)

$\mathrm{Al}$ (arcopallum intermedium)

AM (arcopallium mediale)

$\mathrm{APH}$ (area parahippocampalis)

BaS (basorostral pallial nucleus)

CDL (area corticoidea dorsolateralis)

CPi (cortex piriformis)

HA (hyperpallium apicale)

HD (hyperpallium densocellulare)

HI (hyperpallium intercalatum)

Hp (hippocampus)

HV (hyperpallium ventrale)

MD (mesopallium dorsale)

MV (mesopallium ventrale)

NCL (nidopallium caudolaterale)

NCV (nidopallium caudoventrale)

SL (nucleus septalis lateralis)

TnA (nucleus taeniae amygdalae)

TPO (area temporo-parieto-occipitalis)

Tuo (tuberculum olfactorium)

$+$

$++$

Ipsilateral Contralateral Ipsilateral Contralateral fibers and terminals

$+\quad-\quad+$

$\begin{array}{llll}+++ & +++ & +++ & +++ \\ ++ & ++ & +++ & +\end{array}$

++ $\quad++\quad+++\quad+$

$\begin{array}{llll}- & - & ++\end{array}$

$\begin{array}{llll}++ & +++ & +++ & +++\end{array}$

$+++\quad+++\quad+++\quad++$

$\begin{array}{llll}++ & +++ & +++ & +++\end{array}$

$\begin{array}{llll}++ & + & +++ & ++\end{array}$

$+\quad-\quad+$

$++\quad++\quad+++$

$\begin{array}{llll}- & - & + & +\end{array}$

$+++\quad+++\quad+++$

+++ $\quad+++\quad+++\quad++$

$+++\quad+++\quad+++$

$+++\quad+++\quad++$

++ $\quad$ ++ $\quad++\quad+$

$+\quad+++\quad+$

$\begin{array}{llll}+++ & - & +++ & - \\ - & - & ++ & ++\end{array}$

Diencephalon

Distribution of Labeling of BDA and CTB Injection in PoAb (Table 1 continued)

DMA (nucleus dorsomedialis anterior thalami) 
DMP (nucleus dorsomedialis posterior thalami)

nIV (nucleus nervi trochlearis)

LHy (lateral hypothalamic nucleus)

POA (nucleus preopticus anterior)

POM (nucleus preopticus mediali)

Rt (nucleus rotundus)

RxVM (radix mesencephalicus nervi trigemini)

TeO (tectum opticum)

$++\quad++$

$++$

$++$

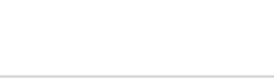

$-$

$+$

$++\quad++$

$+$

$++$

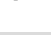

$++$

$++$

$++$

$++$

Distribution of Labeling of BDA and CTB Injection in PoAb (Table. 1 continued)

FLM (fasciculus longitudinalis medialis)

FRM (formatio reticularis medialis mesencephali)

GCt (substantia grisea centralis)

Ico (nucleus intercollicularis)

Imc (nucleus isthmi, pars

magnocellularis)

Ipc (Nucleus isthmi, pars parvocellularis)

LoC (locus ceruleus)

OM (tractus occipito-mesencephalicus)

PL (nucleus pontis lateralis)

PT (nucleus pretectalis)

SAC (stratum album central)

SGC (stratum griseum central)

SGF (stratum griseum et fibrosum superficia)

SGP (substantia grisea et fibrosa periventricularis)

Sop (stratum opticum)

SP (nucleus subpretectalis)

\begin{tabular}{|c|c|c|c|}
\hline - & - & +++ & +++ \\
\hline+++ & +++ & - & - \\
\hline++ & ++ & ++ & ++ \\
\hline++ & - & - & - \\
\hline+++ & +++ & ++ & ++ \\
\hline+++ & +++ & ++ & ++ \\
\hline+ & + & ++ & ++ \\
\hline- & - & ++ & ++ \\
\hline+ & + & - & - \\
\hline- & + & - & - \\
\hline+ & + & ++ & ++ \\
\hline+++ & +++ & +++ & +++ \\
\hline+++ & +++ & +++ & +++ \\
\hline++ & - & ++ & ++ \\
\hline- & - & ++ & ++ \\
\hline+ & + & +++ & +++ \\
\hline
\end{tabular}


SpL (nucleus spiriformis lateralis)

ToS (torus semicircularis)

TVM (tractus vestibulemesencephalicus)

VTA (ventral tegmental area)

$+\quad+$

$+$

$+$

Number of labeled neurons and density of labeled fibers and terminals: +++ , numerous;,++ moderate; + , few; -, absent.

Abbreviations: BDA, biotinylated dextran amine; CTB, cholera toxin subunit B; PoAb, basal division of posterior pallial amygdala

\section{The effects of electrical stimulation on motor behaviors}

Before the electrical stimulus, the pigeons stays in place, generally accompanied by turning its head, or raising its head or pecking feathers in the arena. A series of new different motor behaviors were induced after the electrical stimulation of PoA, among which ipsilateral lateral movement, forward movement, contralateral lateral movement, backward movement, etc., occurred more frequently (Figure 4a, 4d). The different motor behaviors elicited in the arena were defined using a previous study ${ }^{14}$ with slight modifications (see Table2 for details). Figure 4c showed that PoAb mainly mediated ipsilateral lateral movement (75.00\%) and seldomly mediated contralateral lateral movement (1.85\%). PoAc was more complex; the behavioral response of it was as follows: ipsilateral lateral movement (78.89\%), contralateral lateral movement (3.89\%), forward movement (1.11\%), and backward movement (2.78\%). The result indicated that PoA mainly mediated the turning movement behavior in pigeons. There was no significant difference in the ipsilateral lateral movement response rate between PoAc and PoAb (Figure 4f). The locations of the stimuli in PoAc and PoAb are presented in Figure 4b and Figure 4e, respectively.

Table 2

Definitions of some motor behaviors in the arena

\begin{tabular}{|ll|}
\hline Motor behaviors & Definition of the motor behaviors \\
\hline Ipsilateral lateral movement & $\begin{array}{l}\text { Alternate stepping of both feet towards right } \\
\text { side of the body }\end{array}$ \\
\hline Forward movement & Alternate stepping of both feet and moving forward \\
\hline Contralateral lateral movement & Alternate stepping of both feet towards left side of the body \\
\hline Backward movement & Alternate stepping of both feet and moving backward \\
\hline The number of steps generated by different behaviors is generally 2-3 steps. \\
\hline
\end{tabular}




\section{The effects of different drugs on forward behavior}

Personalized screening. According to the SMART output data, the total scores of 25 pigeons were obtained. A normality test was performed on the total scores of each pigeon, and the distribution was found to be non-normal ( $P>0.05, N=25$, Figure 5a). Eight pigeons did not enter the central area (the total score was 0.00 ) (Figure $5 b$ ), and nine pigeons were very active in the central area (the total score was $>1.50$, Figure $5 \mathrm{~d}$ ). Combining the locomotion trajectories of the pigeons in the experimental box captured by SMART, we finally selected those individuals of which the total scores between 0.90 and 1.50. Consequently, eight pigeons were used for the drug injection experiment (Figure $5 c, 5 e)$.

The effects of D1 and D2 receptor agonists and antagonists on the latency of entering central area in pigeons. The effects of D1 and D2 receptor agonists and antagonists on the latency period of pigeons entering the central area were different (Figure 5). D1 agonist decreased the latency period of pigeons for entering the central area $\left(F_{3,28}=14.78, P<0.001\right.$, Figure $\left.5 f\right)$. When the concentration of D1 agonist was $0.5 \mu \mathrm{g} / \mu \mathrm{L}$, the range of the latency period of pigeons for entering the central area decreased by $44.61 \%(P<0.001)$, but there was no significant difference in the other groups. In contrast, $D 1$ antagonist increased the latency period of pigeons for entering the central area $\left(F_{3,28}=\right.$ 27.58, $P<0.001$, Figure $5 \mathrm{~g}$ ). When the concentration of D1 antagonist was $0.1 \mu \mathrm{g} / \mu \mathrm{L}$ and $0.5 \mu \mathrm{g} / \mu \mathrm{L}$, the latency period increased significantly, and their ranges increased by $35.46 \%(P<0.01)$ and $71.61 \%(P<$ $0.001)$, respectively. For $0.02 \mu \mathrm{g} / \mu \mathrm{L}$ concentration of D1 antagonist, the latency period did not increase significantly. D2 agonist also increased the latency period of pigeons for entering the central area $\left(\mathrm{F}_{3,28}=\right.$ $25.63, P<0.001$, Figure $5 \mathrm{~h})$. The ranges of the latency period increased by $45.24 \%(P<0.001)$ and 81.40\% $(P<0.001)$ when the concentrations of D2 agonist were $0.1 \mu \mathrm{g} / \mu \mathrm{L}$ and $0.5 \mu \mathrm{g} / \mu \mathrm{L}$, respectively. However, $0.02 \mu \mathrm{g} / \mu \mathrm{L}$ D2 agonist exhibited no significant influence. D2 antagonist decreased the latency period of pigeons for entering the central area $\left(F_{3,28}=7.74, P<0.001\right.$, Figure 5i). The latency period of pigeons for entering the central area was decreased by $26.46 \%(P<0.001)$ when the concentration of D2 antagonist was $0.02 \mu \mathrm{g} / \mu \mathrm{L}$; but when the concentrations of D2 antagonist were 0.1 $\mu \mathrm{g} / \mu \mathrm{L}$ and $0.5 \mu \mathrm{g} / \mu \mathrm{L}$, the latency period was decreased by $16.69 \%(P<0.05)$ and $18.89 \%(P<0.05)$, respectively. Overall, D1 agonist and D2 antagonist can reduce the latency period of pigeons for entering the central area. In contrast, D1 antagonists and D2 agonists could increase the latency period of pigeons for entering the central area.

The effects of D1 and D2 receptor agonists and antagonists on the stay time of pigeon in the central area. D1 and D2 receptor agonists and antagonists have different effects on the stay time of pigeons in the central area (Figure 5). D1 agonist increased the stay time of pigeons in the central area $\left(F_{3,28}=\right.$ $11.59, P<0.001$, Figure $5 \mathrm{j}$ ). When the concentration of $\mathrm{D} 1$ agonist was $0.1 \mu \mathrm{g} / \mu \mathrm{L}$ and $0.5 \mu \mathrm{g} / \mu \mathrm{L}$, the stay time of pigeons in the central area increased by $58.12 \%(P<0.001)$ and $62.03 \%(P<0.001)$, respectively, 
but when the concentration of D1 agonist was $0.02 \mu \mathrm{g} / \mu \mathrm{L}$, no significant difference was noted in the stay time. D1 antagonist decreased the stay time of pigeons in the central area $\left(F_{3,28}=26.19, P<0.001\right.$, Figure $5 \mathrm{k})$. The stay time of pigeons in the central area decreased by $40.21 \%(P<0.001)$ when the concentration of D1 antagonist was $0.1 \mu \mathrm{g} / \mu \mathrm{L}$. D2 agonist had a significant impact on the stay time of pigeons in the central area $\left(F_{3,28}=34.64, P<0.001\right.$, Figure $\left.5 \mathrm{l}\right)$. When the concentration of D2 agonist was $0.02 \mu \mathrm{g} / \mu \mathrm{L}$, the stay time in the central area increased by $53.72 \%(P<0.001)$; whereas it decreased by $12.53 \%$ when the concentration of D2 agonist was $0.5 \mu \mathrm{g} / \mu \mathrm{L}$, and no significant difference was noted. D2 antagonist increased the stay time of pigeons in the central area $\left(F_{3,28}=81.74\right.$, $P<0.001$, Figure $5 \mathrm{~m})$. The stay time of pigeons in the central area increased by $25.92 \%(P<0.01)$ and 92.19\% $(P<0.001)$ when the concentration of D2 antagonist was $0.1 \mu \mathrm{g} / \mu \mathrm{L}$ and $0.5 \mu \mathrm{g} / \mu \mathrm{L}$, respectively; however, $0.02 \mu \mathrm{g} / \mu \mathrm{L}$ D2 antagonist did not result in significant changes in the stay time. In summary, with increase in drug concentration, D1 agonist and D2 antagonist significantly increased the stay time of pigeons in the central area. In contrast, D1 antagonist decreased the stay time of pigeons in the central area. Further, D2 agonist increased the stay time of pigeons in the central area at a low concentration but decreased the stay time at a high concentration.

\section{Discussion}

The present study revealed the fiber connections of PoAb, which innervate most of the telencephalon, especially the pallium. Concerning descending pathways, PoAb projects primarily to the midbrain. The labeling areas are consistent with those reported by previous researchers in forebrain ${ }^{6,8-9,15-20}$. Compared with PoAb, PoAc has few connections with the pallium. As for the downstream pathways, PoAc connects primarily with the diencephalon and projects less on the lateral hypothalamus. The connections between PoAb and pallium were confirmed in pigeons, which are in accordance with the findings in chickens or mallards, in spite of the projections between PoA and the ventral basal ganglia or medial striatum have not been proven in chickens or mallards ${ }^{21-22}$. However, the tectal layers and the associated isthmic complex were not described ascending projections to the pallium in other studies. But the present study show that PoAb connects reciprocally with SGC, SGF, and SAC and so on. This may be caused by different development process methods or false positive findings. And we did not draw the controversial brain areas in the summary diagram. As the second major vertebrates, birds have no corpus callosum (CC). The connection of PoAb with many areas in the brain of pigeons suggests that it plays an essential role in interhemispheric exchange, which consequently indicates that PoAb compensates for the absence of $\mathrm{CC}$ in birds.

The results of electrical stimulation indicated that PoAb can single-handedly mediate ipsilateral lateral movement, whereas PoAc is different. The differences in PoAb and PoAc with regard to connectivity result in differences in their mediation of motor behaviors. PoAb has closer connections with pallium than does PoAc. As for the descending pathways, PoAb connects with many locomotor brain regions in the midbrain, whereas PoAc has little connections with locomotor brain regions in the midbrain, most of which are one-way projections. PoAc has significantly more connections with other brain regions within 
the amygdala of birds than does PoAb ${ }^{8}$. Perhaps the close connections within the amygdala lead to the complexity of PoAc behavior. Moreover, the molecular mechanisms of PoAb and PoAc on the mediation of motor behavior in pigeons remain to be studied.

Each pigeon's total score exhibited whether the activity time and activity degree of the pigeon in the central area were random or not. Compared with mice, pigeons have poor ability to explore new environments; thus, the total scores of most pigeons were 0.00 , and only few pigeons were very active. The reason for the significant difference may be that the brains of birds and mammals have evolved differently.

The effects of different drugs on forward behavior revealed that D1 agonist and D2 antagonist could promote forward behavior, whereas D1 antagonist and D2 agonist have inhibitory effects. Our results corroborated those of previous studies in Wistar rats, hens, and Japanese quail ${ }^{11,23-25}$. However, compared with reptiles, the effects of D1 and D2 receptor agonists and antagonists were partly in accordance with those on pigeons ${ }^{12,26-27}$; this could be attributed to the difference in species. Since experiments in this part mainly analyze the movement of pigeons, the forward behavior was directly related to the pigeon movement. Besides, PoAc was characterized by high tyrosine hydroxylase (TH) immunoreactivity, whereas PoAb has negligible numbers of $\mathrm{TH}$ positive cells ${ }^{7,9}$. Thus, we chose the dominant site of forward behavior in PoAc for drug injection. The PoA, TnA, area subpallialis amygdalae $(\mathrm{SpA})$, bed nucleus of the stria terminalis, and pars lateralis (BSTL) were considered to form the avian amygdala ${ }^{7}$. Dopamine plays an important role in the regulation of emotions ${ }^{28}$. By comparing the behavioral differences between the experimental group and the control group, the role of PoAc in the regulation of forward behavior is studied. The results of this part further prove that PoAc affects the behavior of pigeons by regulating their emotions.

Overall, PoAb connects with many more areas in the cerebrum than PoAc, suggesting that the role of PoAb was exchanged between the right and left hemispheres. The function of PoAb and PoAc is to mediate the turning behavior, and both of them can be used as important brain areas to regulate turning behavior. The effects of different drugs on forward behavior proved that PoAc affects motor behaviors by regulating emotion. Our study will provide a theoretical basis for controlling motor behavior in birds and enrich the theoretical system of emotion-related brain regions and behaviors in birds.

\section{Methods}

\section{Animals.}

Overall, 65 adult pigeons (body mass, $350-450 \mathrm{~g}$ ) were used in this study. All animals (unknown sexes) were acclimatized to a wire cage $\left(95 \times 80 \times 65 \mathrm{~cm}^{3}\right)$ under a normal day/night light cycle with food and water ad libitum. The feedstuff was composed of wheat, corn, sorghum, and soybean at the ratio of 1:1:1:1, and the cages were cleaned twice a week. All experimental procedures and animal housing and manipulations were approved by and carried out under the Life Science Ethical Review Committee of 
Zhengzhou University (Henan, China). All efforts were made to minimize the suffering of the animals throughout the experimental procedures.

Declarations: The study was carried out in compliance with the ARRIVE guidelines, and all methods were carried out in accordance with relevant guidelines and regulations.

\section{Tract-tracing of PoAb pathway}

Surgical procedure. The pigeons were anesthetized by injecting $3 \%$ pelltobarbitalum natricum $(0.12$ $\mathrm{ml} / 100 \mathrm{~g}$ body mass) intraperitoneally. After the pain reflex disappeared, the pigeons' heads were placed in a stereotaxic holder customized for the pigeons. The skin above the surgical field was removed carefully, part of the skull was removed using a dental drill, the dura mater and arachnoid mater were moved, and the brain tissue was exposed. Subsequently, the injection cannula (O.D.0.41 × I.D.0.25; R.W.D. Life Science, Shenzhen, China) was implanted. When the PoAb was reached, the gaps between the cannula and the cranium were sealed with EC glue and fixed with dental cement after the glue solidified. Enrofloxacin solution (5\%) was used in the surgical area to help recovery. The surgery area was cleaned regularly. The pigeons were fed separately during 5-7 days of recovery, after which they were used in the following experiments.

In vivo injections. After recovery, each pigeon received unilateral injections of one tracer, BDA (10,000 molecular weight; Thermo Fisher, Eugene, OR, USA) or CTB (low salt; List Biological Laboratories, Campbell, CA, USA) on the right hemisphere (Figure 1a, 1f). BDA (10\% in phosphate-buffered saline [PBS], $\mathrm{pH}$ 7.4) was used for anterograde labeling, whereas CTB (1\% in PBS) was used for retrograde labeling. At each injection site, $150 \mathrm{nl}$ of the tracer was applied at each depth using a mechanical pressure device (R.W.D. Life Science, Shenzhen, China). After 2 days (for CTB injections) or 7 days (for BDA injections) of survival, the pigeons were deeply anesthetized using pelltobarbitalum natricum $\left(100 \mathrm{mg} \cdot \mathrm{kg}^{-1}\right)$ and perfused with physiological salt solution, followed by cardiac perfusion with $4 \%$ formaldehyde.

Their brains were extracted and postfixed overnight in $4 \%$ paraformaldehyde at $4^{\circ} \mathrm{C}$ and subsequently transferred to $30 \%$ sucrose in PBS at $4^{\circ} \mathrm{C}$ for 3 days. Brains were cut at the coronal plane in 35- $\mu \mathrm{m}$ thick slices using a freezing microtome (Leica CM1950, Germany).

Immunohistochemistry. BDA and CTB fluorescence staining was used to demonstrate anterogradely labeled fibers and retrogradely labeled neurons.

For CTB fluorescence staining, slices were washed with PBS before $(3 \times 10 \mathrm{~min})$ and after $(1 \times 5 \mathrm{~min})$ incubation in 10\% normal goat serum (30 min; 1:10 in PBS). Subsequently, they were incubated with polyclonal rabbit anti-CTB antibody (Abcam, RRID: AB_34992; 1:1,000 in PBS) for $12-14 \mathrm{~h}$ at $4{ }^{\circ} \mathrm{C}$. Then, the slices were washed in PBS $(3 \times 10 \mathrm{~min})$ and incubated with the secondary antibody Alexa Fluor ${ }^{\circledR}$ 488-conjugated AffiniPure Goat Anti-Rabbit IgG (Wuhan service biotechnology; 1:200 in PBS) for 1 $\mathrm{h}$ at $37^{\circ} \mathrm{C}$. Finally, the slices were washed in PBS $(3 \times 10 \mathrm{~min})$, mounted on glass slides, and covered using a coverslip with fluoromount (Wuhan service biotechnology, Wuhan, China). 
For BDA fluorescence staining, after the slices were incubated for 30 min in 10\% normal goat serum, they were incubated with Alexa 594 streptavidin (BioLegend Way San Diego, CA; 1:200 in PBS) for 50 min at $37^{\circ} \mathrm{C}$. Finally, the slices were washed in PBS $(3 \times 10 \mathrm{~min})$, mounted on glass slides, and covered using a coverslip with fluoromount.

\section{The effects of PoA electrical stimulation on motor behaviors}

Microelectrode implants. The electrode (diameter $=120 \mu \mathrm{m}$; stainless steel wire, Teflon insulation) was implanted into PoAb and PoAc, respectively $(N=3)$. All processes were performed as described above.

Electrical stimulation protocols. After recovery, the behavioral responses of the pigeons to electrical stimulation were observed. The subjects were given $5 \mathrm{~min}$ for adaptation, and then electrical stimulation (stimulation intensity $=0.3-0.8 \mathrm{~mA}$; interstimulus interval $=3-5 \mathrm{~min}$; frequency $=20 \mathrm{~Hz}$ ) generated by an YC - 2 - S bipolar programmed stimulator (Chengdu Instrument Factory, China) was applied via stainless steel electrodes (diameter $=120 \mu \mathrm{m}$; impedance range < $0.5 \mathrm{M} \Omega$ ). The test was done in an arena made of polymathic methacrylate (diameter $\times$ height, $80 \times 80 \mathrm{~cm}^{2}$ ). The responsive behaviors evoked by different stimulation intensities were recorded using a SONY HDR-CX220 camcorder (Sony Corporation, Tokyo, Japan).

Histology. After three test trials (inter-experiment interval was 3 days), the pigeons were deeply anesthetized and then perfused. Subsequently, their brains were removed from the skulls, postfixed, and dehydrated. Lastly, histological analyses were performed by Nissl and hematoxylin and eosin staining. Light microscopy was used to identify the stimulation sites of the sections.

\section{The effects of different drugs on forward behavior}

Personalized screening. The pigeons should be screened to avoid the impact of personalized differences before the experiment. According to the method used for studying jungle crow's response to mirror-image stimulation ${ }^{10}$, the size of the experimental box was $100 \times 100 \times 40 \mathrm{~cm}^{3}$ (length $\times$ width $\times$ height), and the video equipment was installed $120 \mathrm{~cm}$ above the experimental box. The experimental box was divided into two areas: the central area (within the red box) and the edge area (outside the red box). The central area was square with an area of $33.33 \times 33.33 \mathrm{~cm}^{2}$, accounting for $1 / 9$ of the whole area.

The pigeons were placed in the edge area of the experimental box when the recording device was working. Each pigeon was recorded for $15 \mathrm{~min}$. The first $5 \mathrm{~min}$ recorded the pigeons' adaptation process to the experimental box, and the last 10 min constituted the actual personalized screening videotape. Subsequently, 75\% alcohol was used to spray the whole experimental box, and the next experiment was recorded after $30 \mathrm{~min}$. SMART 3.0 Panlab video analysis software (Henan Baiquan Bioscientific Co.,limited, China) was used to collect the data on movement behavior of each pigeon ( $N=$ 
25) automatically; the data mainly included movement time, movement speed, and movement distance of the pigeon in various areas of the experimental box; the movement trajectory of the pigeon was also captured. The personality screening criteria were determined by the movement time, distance, and activity degree in each area of the experimental box. The personality score of each pigeon was calculated as follows: (movement distance in the central area $\times 9$ / total distance) / (movement time in the central area $\times 9$ / total time). The pigeon motion trajectory map obtained by SMART software was used on an auxiliary basis.

Stereotaxic implantation of the injection cannula. After personalized screening, the pigeons $(N=8)$ were implanted with an injection cannula (0.D.0.41 x I.D.0.25) at the site of PoAc (A $5.00 \mathrm{~mm} ; \mathrm{L} 7.60 \mathrm{~mm}$ ). All processes were performed as described above.

Drug treatments. After cannula implantation, the recovered pigeons were administered four different drugs ( $N=8$ ): SKF-38393 (D1 +: D1 agonist), SCH-23390 (D1 -: D1 antagonist), quinpirole (D2 +: D2 agonist), and raclopride (D2 :- D2 antagonist); all drugs were bought from Sigma-Aldrich (St louis, USA). According to the methods in Japanese quail ${ }^{11}$ and male green anoles ${ }^{12}$, three concentration gradients were set for each drug: $0.02 \mu \mathrm{g} / \mu \mathrm{L}$ (low), $0.1 \mu \mathrm{g} / \mu \mathrm{L}$ (medium), $0.5 \mu \mathrm{g} / \mu \mathrm{L}$ (high); $0.9 \%$ saline was used as the control. Because the four drugs can be metabolized autonomously in pigeons, two injections can be performed for each drug.

First, the pigeons in the control group were injected $1 \mu \mathrm{L}$ of saline, and their movement behaviors were recorded in the experimental box for $30 \mathrm{~min}$; then the pigeons were injected $1 \mu \mathrm{L}$ of 0.02 $\mu \mathrm{g} / \mu \mathrm{L}$ D1 agonist (low concentration), and their movement behaviors were recorded for $30 \mathrm{~min}$ after 15 min of drug absorption time. After 1 day, $1 \mu \mathrm{L}$ of saline was injected as control, following which $1 \mu \mathrm{L}$ of $0.1 \mu \mathrm{g} / \mu \mathrm{L}$ D1 agonist (medium concentration) was injected; after $15 \mathrm{~min}$, their movement behaviors were recorded for $30 \mathrm{~min}$. Another day later, $1 \mu \mathrm{L}$ of saline was injected as control, and then 1 $\mu \mathrm{L}$ of $0.5 \mu \mathrm{g} / \mu \mathrm{L}$ D1 agonist (high concentration) was injected; after 15 min, their movement behaviors were recorded for $30 \mathrm{~min}$. Different concentrations of D1 antagonist, D2 agonist, and D2 antagonist were injected; the experimental procedure was the same as that for D1 agonist, and the interval between the injection of different drugs was 4 days.

\section{Data recording and analysis.}

The terminology used in the present study is based on the Avian Brain Nomenclature Forum ${ }^{1,13}$.

Photomicrographs were captured using a digital camera (Olympus, Japan). The layout of micrographs, lettering, and printing, and schematic illustrations of labeled neurons and fibers were performed using Adobe Photoshop 7.0J (Tokyo, Japan) and Adobe Illustrator 10.0J (Tokyo, Japan).

All data were analyzed on IBM SPSS Statistics 24 (Chicago, USA). Data were recorded on SMART 3.0 software, which included all relevant data and animal behavior trajectory data. The data standardization 
method was as follows: for each animal, (data of each treatment/sum of four treatments) $\times 100 \%$. The Kolmogorov-Smirnov test was used to test normality of the data, and one-way ANOVA was used to analyze the drug administration data. Data of all groups are represented as mean \pm standard error of the mean.

\section{Declarations}

\section{Acknowledgements}

This work was supported by the National Natural Science Foundation of China, Grant No. U2004152.

\section{Authors' contributions}

XM Tian collected and analyzed data and wrote the initial draft of the paper. YH Shi collected the data. YF Zhang analyzed the data. YJ Wang and MK Li were responsible for image processing. $\mathrm{H}$ Cheng and ZL Wang planned the experiments, analyzed the data, and helped write the paper.

\section{Conflicts of Interest}

The authors declare that they have no conflict of interest.

\section{Peer Review}

The peer review history for this article is available at https://www.researchsquare.com/article/rs$620016 / v 1$.

\section{References}

1. Reiner, A. et al. Revised nomenclature for avian telencephalon and some related brainstem nuclei. J. Comp. Neurol, 473, 377-414 (2004).

2. Dafters, R. Active avoidance behavior following archistriatal lesions in pigeons. J. Comp. Psychol, 89, 1169-1179 (1975).

3. Lowndes, M. \& Davies, D. C. The effect of archistriatal lesions on'open field'and fear/avoidance behaviour in the domestic chick. Behav. Brain Res, 72, 25-32 (1995).

4. Phillips, R. E. \& Youngren, O. M. Unilateral kainic acid lesions reveal dominance of right archistriatum in avian fear behavior. Brain Res, 377, 216-220 (1986).

5. Saint-Dizier, H. et al. Subdivisions of the arcopallium/posterior pallial amygdala complex are differentially involved in the control of fear behaviour in the Japanese quail. Brain Res. Bull, 79, 288295 (2009). 
6. Zeier, H. \& Karten, H. J. The archistriatum of the pigeon Organization of afferent and efferent connections. Brain Res, 31, 313-326 (1971).

7. Herold, C., Paulitschek, C., Palomero-Gallagher, N., Gunturkun, O. \& Zilles, K. Transmitter receptors reveal segregation of the arcopallium/amygdala complex in pigeons (Columba livia). J. Comp. Neurol, 526, 439-466 (2018).

8. Atoji, Y., Saito, S. \& Wild, J. M. Fiber connections of the compact division of the posterior pallial amygdala and lateral part of the bed nucleus of the stria terminalis in the pigeon (Columba livia). $J$. Comp. Neurol, 499, 161-182 (2006).

9. Kroner, S. \& Gunturkun, O. Afferent and efferent connections of the caudolateral neostriatum in the pigeon (Columba livia): a retro- and anterograde pathway tracing study. J. Comp. Neurol, 407, 228260 (1999).

10. Kusayama, T., Bischof, H. J. \& Watanabe, S. Responses to mirror-image stimulation in jungle crows (Corvus macrorhynchos). Anim. Cogn, 3, 61-64 (2000).

11. Kleitz-Nelson, H. K., Cornil, C. A., Balthazart, J. \& Ball, G. F. Differential effects of central injections of D1 and D2 receptor agonists and antagonists on male sexual behavior in Japanese quail. Eur. J. Neurosci, 32, 118-129 (2010).

12. Smith, A. N. \& Kabelik, D. The effects of dopamine receptor 1 and 2 agonists and antagonists on sexual and aggressive behaviors in male green anoles. PLoS One, 12, 1-10 (2017).

13. Karten, H. J. \& Hodos, W. A stereotaxic atlas of the brain of the pigeon (Columba livia) (Johns Hopkins University Press, Baltimore, 1967).

14. Tian, X. M. et al. The impact of tag position and mass on motor behavior in pigeons. J. Ornithol, 161, 1167-1174 (2020).

15. Atoji, Y. \& Wild, J. M. Afferent and efferent connections of the dorsolateral corticoid area and a comparison with connections of the temporo-parieto-occipital area in the pigeon (Columba livia). $\mathrm{J}$. Comp. Neurol, 485, 165-182 (2005).

16. Atoji, Y. \& Wild, J. M. Afferent and efferent projections of the central caudal nidopallium in the pigeon (Columba livia). J. Comp. Neurol, 517, 350-370 (2009).

17. Atoji, Y. \& Wild, J. M. Afferent and efferent projections of the mesopallium in the pigeon (Columba livia). J. Comp. Neurol, 520, 717-741 (2012).

18. Atoji, Y. \& Wild, J. M. Projections of the densocellular part of the hyperpallium in the rostral Wulst of pigeons (Columba livia). Brain Res, 1771, 130-139 (2019).

19. Letzner, S., Simon, A. \& Gunturkun, O. Connectivity and neurochemistry of the commissura anterior of the pigeon (Columba livia). J. Comp. Neurol, 524, 343-361 (2016).

20. Behroozi, M. et al. Event-related functional MRI of awake behaving pigeons at 7T. Nat. Commun, 11, $1-12(2020)$.

21. Davies, D. C., Csillag, A., Szekely, A. D. \& Kabai, P. Efferent connections of the domestic chick archistriatum: a phaseolus lectin anterograde tracing study. J. Comp. Neurol, 389, 679-693 (1997). 
22. Dubbeldam, J. L., Boer-Visser, A. M. D. \& Bout, R. G. Organization and efferent connections of the archistriatum of the mallard, Anas platyrhynchos L.: an anterograde and retrograde tracing study. $J$. Comp. Neurol, 388, 632-657 (1997).

23. Castagna, C., Ball, G. F. \& Balthazart, J. Effects of dopamine agonists on appetitive and consummatory male sexual behavior in Japanese quail. Pharmacol. Biochem. Be, 58, 403-414 (1997).

24. de la Mora, M. P. et al. Anxiolytic effects of intra-amygdaloid injection of the D1 antagonist SCH23390 in the rat. Neurosci. Lett, 377, 101-105 (2005).

25. Dennis, R. L. \& Cheng, H. W. The dopaminergic system and aggression in laying hens. Poultry Sci, $\mathbf{9 0 ,}$ 2440-2448 (2011).

26. Woolley, S. C., Sakata, J. T., Gupta, A. \& Crews, D. Evolutionary changes in dopaminergic modulation of courtship behavior in Cnemidophorus whiptail lizards. Horm. Behav, 40, 483-489 (2001).

27. Woolley, S. C., Sakata, J. T. \& Crews, D. Evolutionary insights into the regulation of courtship behavior in male amphibians and reptiles. Physiol. Behav, 83, 347-360 (2004).

28. de la Mora, M. P., Cárdenas-Cachón, L., Arizmendi-Garica, Y., Marcellino, D. \& Fuxe, K. Role of dopamine receptor mechanisms in the amygdaloid modulation of fear and anxiety: Structural and functional analysis. Prog. Neurobiol, 90, 198-216 (2010).

\section{Figures}
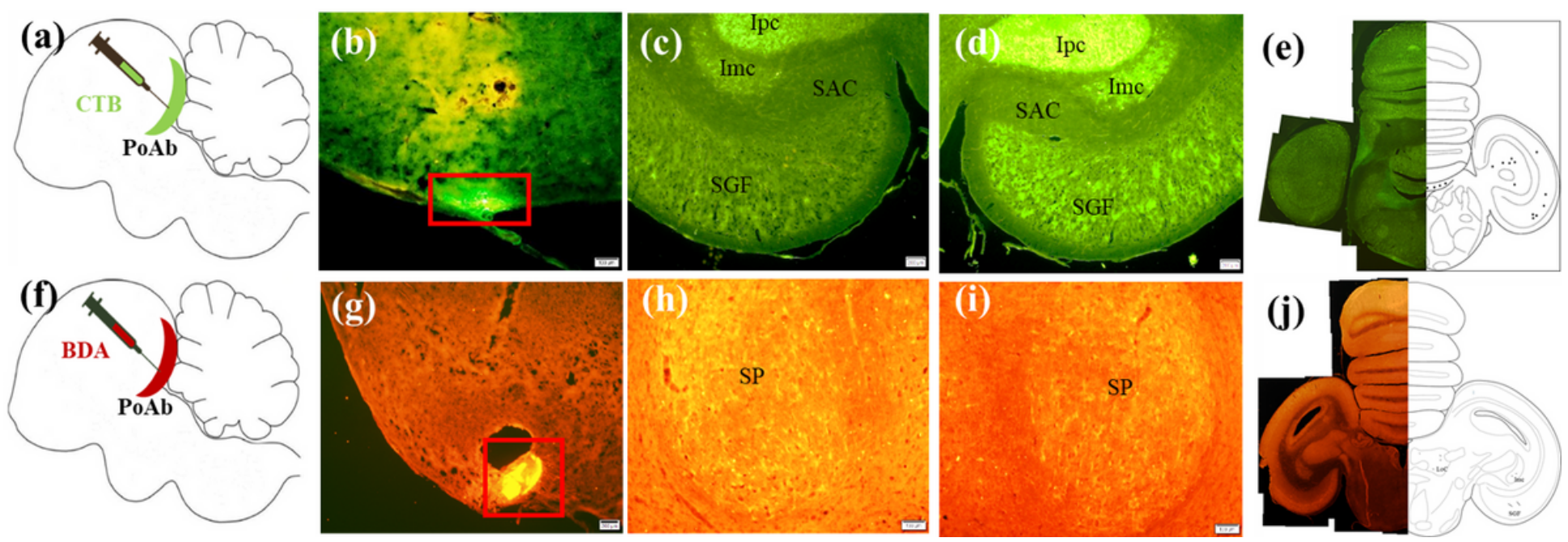

\section{Figure 1}

Fiber connections of PoAb. Schematic of in vivo CTB injections (a) and BDA injections ( $f$ ) into PoAb. (b): Injection site of CTB in the PoAb at A 6.25. (c): Ipsilateral CTB-labeled neurons in Imc at A 3.5. (d): Contralateral CTB-labeled neurons in Imc at A 3.5. (e): Schematic of CTB-labeled at A 1.25. (g): Injection site of BDA in the PoAb at A 5.75. (h): Ipsilateral labeled fibers in SP at A 5.25. (i): Contralateral labeled fibers in SP at A 5.25. (j): Schematic of BDA-labeled at A 2.75. Scale bars $=200 \mu \mathrm{m}$ in (c), (d), (g); $100 \mu \mathrm{m}$ 
in (b), (h), (i). Abbreviations: BDA, biotinylated dextran amine; CTB, cholera toxin subunit B; Imc, nucleus isthmi, pars magnocellularis; Ipc, nucleus isthmi, pars parvocellularis; PoAb, basal division of posterior pallial amygdala; SAC, stratum album central; SGF, stratum griseum et fibrosum superficial; SP, nucleus subpretectalis
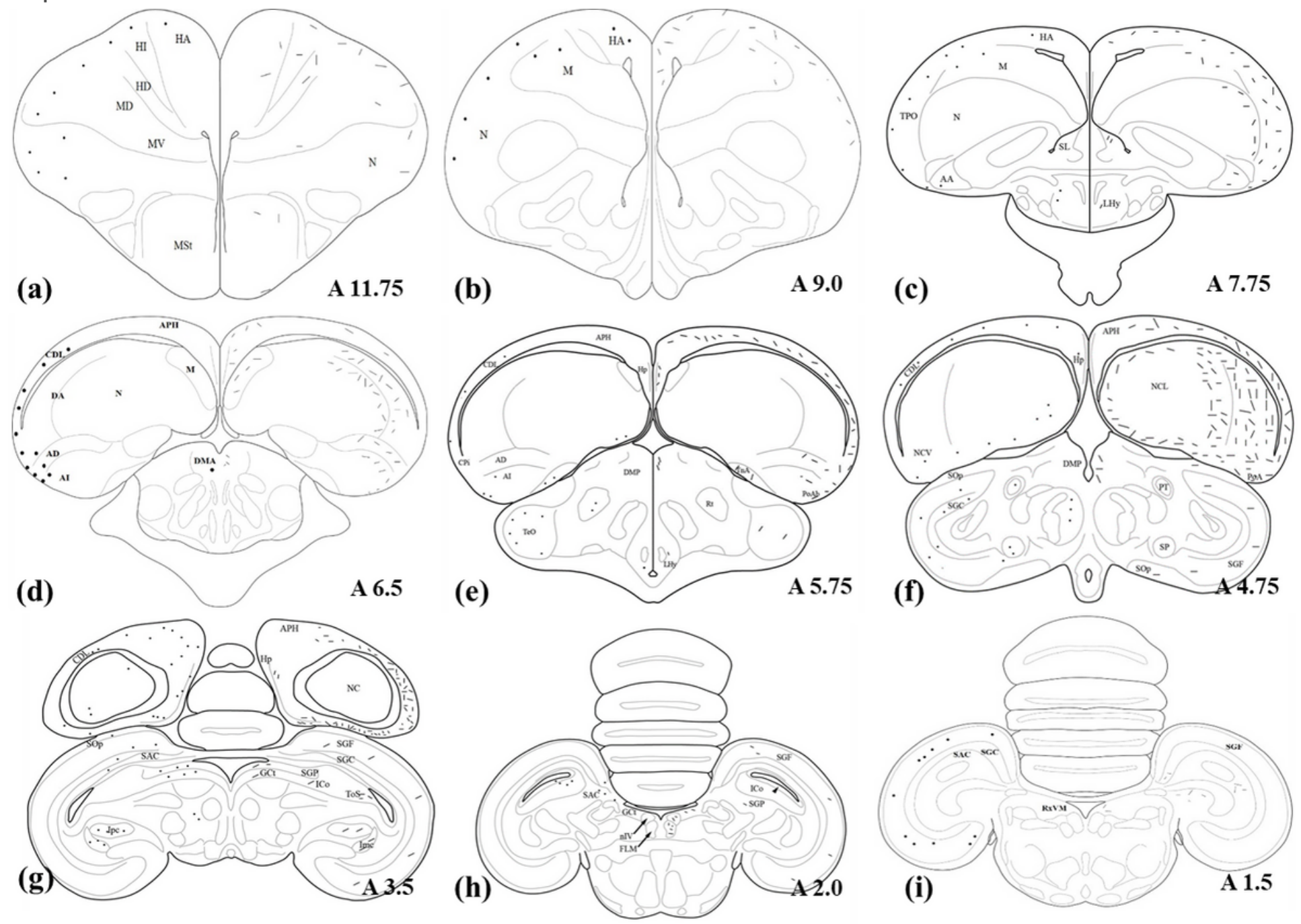

Figure 2

Schematic illustration of the rostrocaudal extent of labeling after BDA or CTB injections into the PoAb. Dots represent neurons retrogradely labeled with CTB on the left side, short lines represent anterogradely labeled terminals on the right side. Dots and lines indicate the relative differences in distribution but do not represent actual numbers. Abbreviations: BDA, biotinylated dextran amine; CTB, cholera toxin subunit $B$; PoAb, basal division of posterior pallial amygdala 


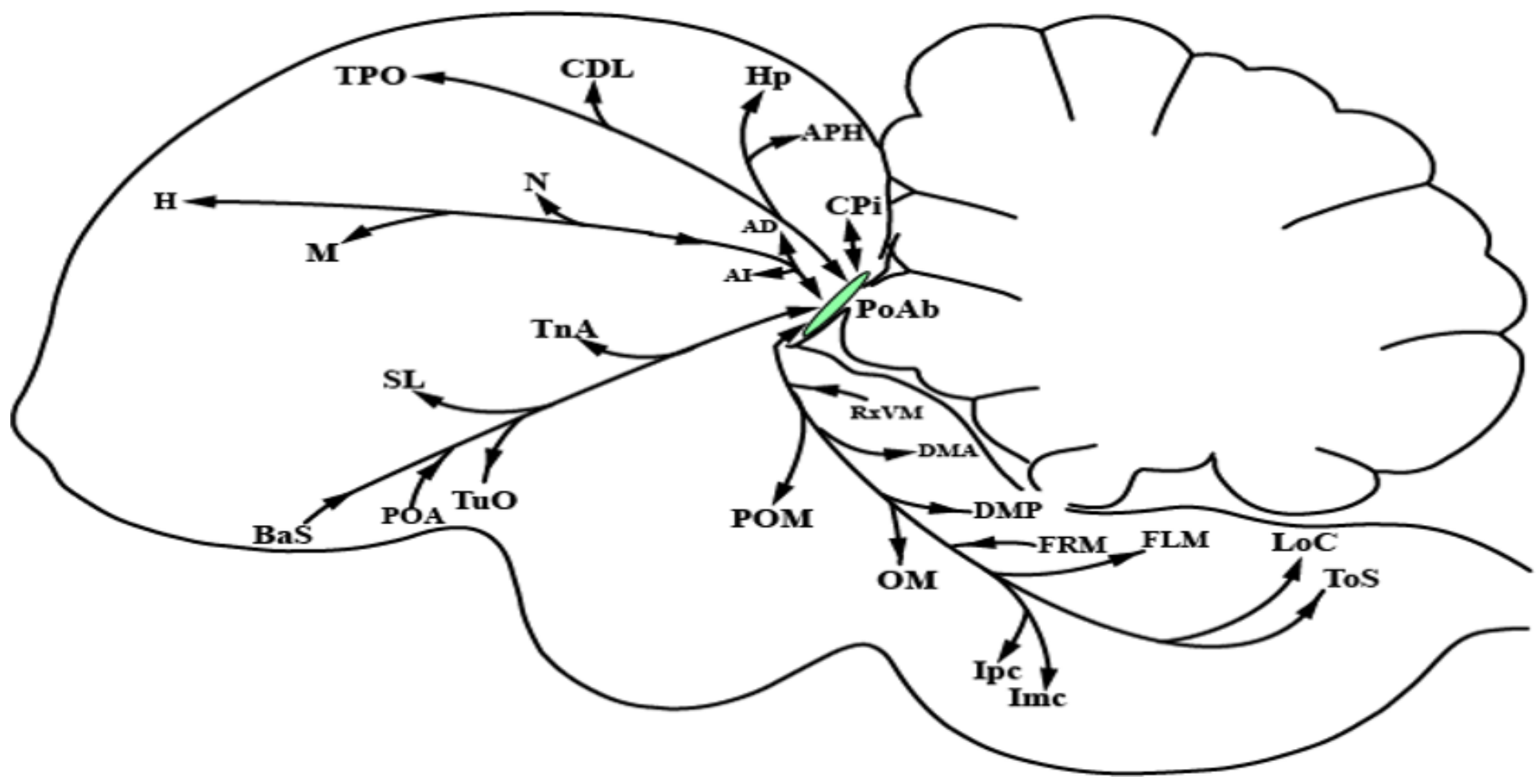

Figure 3

Models of main pathways of PoAb in the pigeon brain. Arrowheads at end of arrows show reciprocal connections, whereas those present midway indicate one-way projections. Abbreviations: $A D$, arcopallium dorsale; Al, arcopallium intermedium; $\mathrm{APH}$, area parahippocampalis; $\mathrm{BaS}$, basorostral pallial nucleus; $\mathrm{CDL}$, area corticoidea dorsolateralis; $\mathrm{CPi}$, cortex piriformis; $\mathrm{DMA}$, nucleus dorsomedialis anterior thalami; DMP, nucleus dorsomedialis posterior thalami; FLM, fasciculus longitudinalis medialis; FRM, formatio reticularis medialis mesencephali; $\mathrm{H}$, hyperpallium; Imc, nucleus isthmi, pars magnocellularis; Ipc, nucleus isthmi, pars parvocellularis; LoC, locus ceruleus; $\mathrm{M}$, mesopallium; $\mathrm{N}$, nidopallium; $\mathrm{OM}$, tractus occipitomesencephalicus; POA, nucleus preopticus anterior; PoAb, basal division of posterior pallial amygdala; POM, nucleus preopticus mediali; RxVM, radix mesencephalicus nervi trigemini; SL, nucleus septalis lateralis; SP, nucleus subpretectalis; TnA, nucleus taeniae amygdalae; TPO, area temporo-parietooccipitalis; Tuo, tuberculum olfactorium 

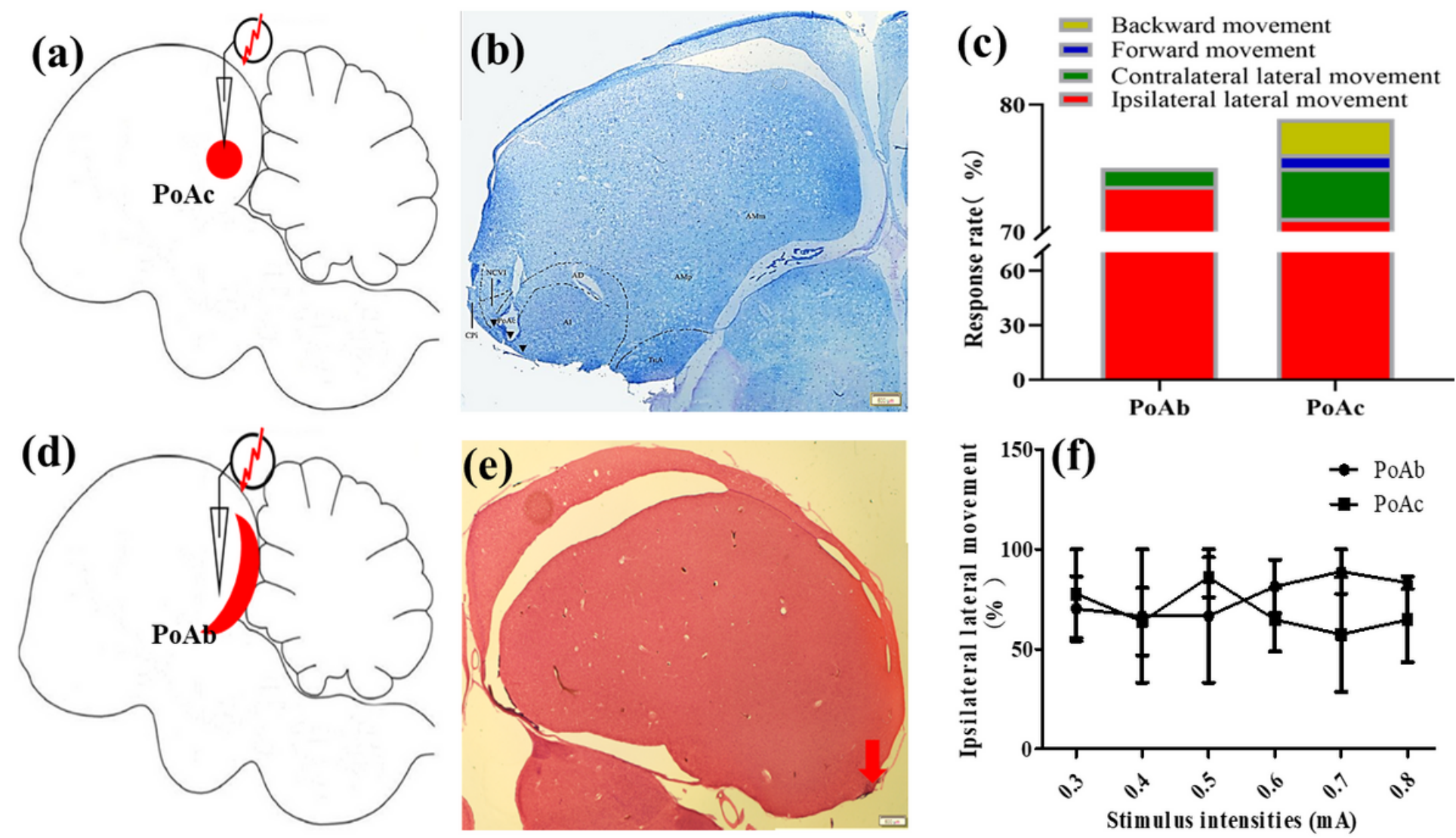

\section{Figure 4}

Effects of electrical stimulation on motor behaviors. Schematic of electrical stimulation of PoAc (a) and PoAb (d). Coronal slice of PoAc Nissl staining at A 5.0 (b) and PoAb hematoxylin and eosin staining at A 5.5 (e). (c): Response rate of four different behaviors after electrical stimulation of PoAc and PoAb. (F): Response rate of ipsilateral lateral movement of PoAc and PoAb at different electrical stimulation intensities. Scale bars $=500 \mu \mathrm{m}$ in (b) and (e). Abbreviations: AD, arcopallium dorsale; $\mathrm{Al}$, arcopallium intermedium; AM, arcopallium mediale; AMm, arcopallium mediale, pars magnocellularis; AMp, arcopallium mediale, pars parvocellularis; $\mathrm{CPi}$, cortex piriformis; NCV, nidopallium caudoventrale; PoAb, basal division of posterior pallial amygdala; PoAc, compact division of posterior pallial amygdala; TnA, nucleus taeniae amygdalae 

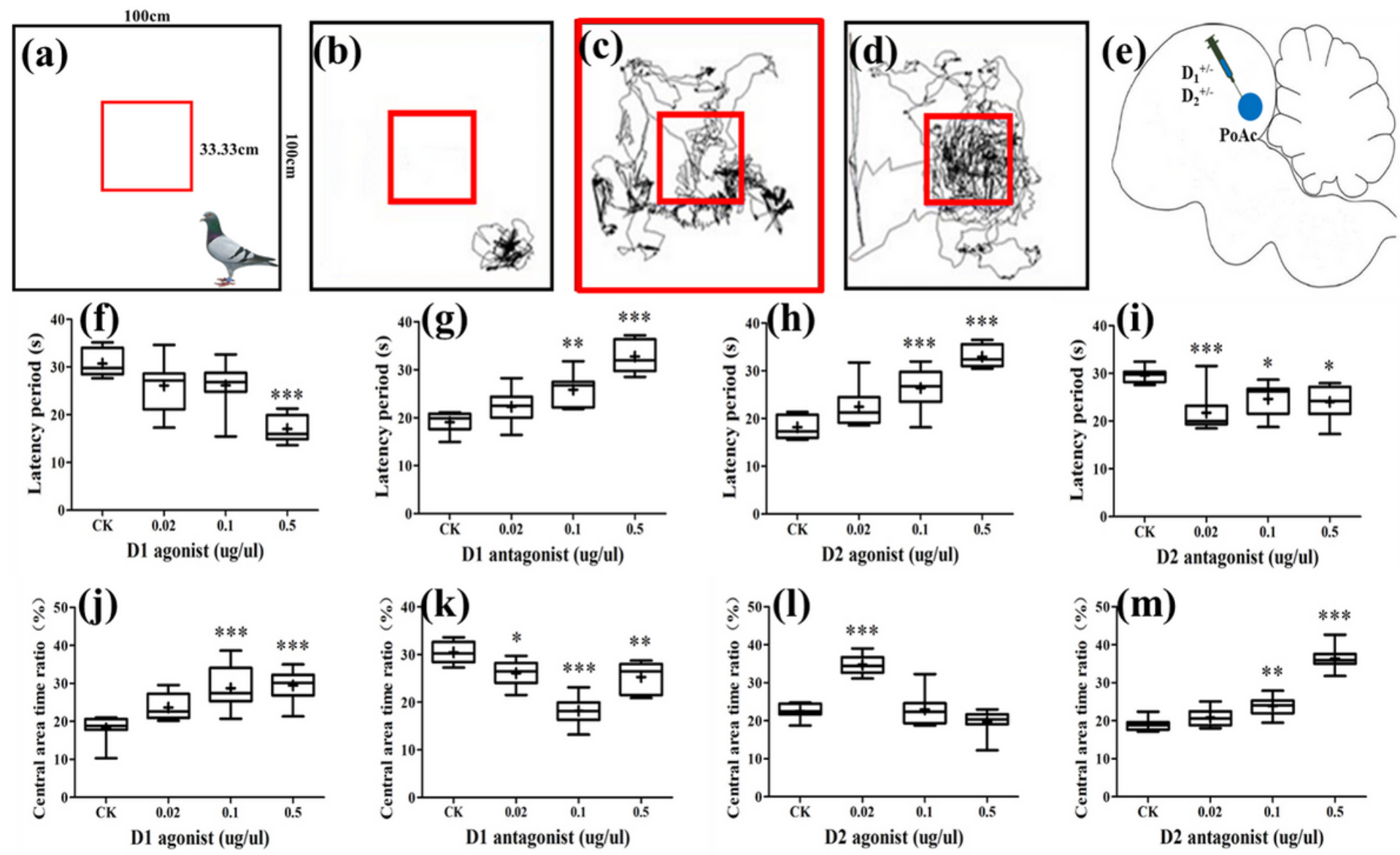

\section{Figure 5}

Effects of different drugs on forward behavior. (a): Schematic of personalized screening. (b-d): Locomotion trajectory of different pigeons. (e): Schematic of in vivo injections of different drugs into PoAc. (f-i): Effects of D1 and D2 receptor agonists and antagonists on the latency period of pigeons for entering the central area. (j-m): Effects of D1 and D2 receptor agonists and antagonists on the stay time of pigeons in the central area. Data of all groups are presented as mean $\pm \mathrm{SE}$. Abbreviations: PoAc, compact division of posterior pallial amygdala; SE, standard error of mean 